\title{
Article
}

\section{The Laplace Transform of Quantum Gravity}

\author{
Jorge Gamboa ${ }^{1, *,+}+\mathbb{D}$, Fernando Méndez ${ }^{1,+}+\mathbb{D}$ and Natalia Tapia-Arellano ${ }^{2,+}+\mathbb{D}$ \\ 1 Departamento de Física, Facultad de Ciencia, Universidad de Santiago de Chile (USACH), \\ Santiago 8320000, Chile \\ 2 Center for Neutrino Physics, Department of Physics, Virginia Tech, Blacksburg, VA 24061, USA; \\ fernando.mendez@usach.cl (F.M.); ntapiaa@vt.edu (N.T.-A.) \\ * Correspondence: jorge.gamboa@usach.cl \\ + These authors contributed equally to this work.
}

check for updates

Citation: Gamboa, J.; Méndez, F.; Tapia-Arellano, N. The Laplace Transform of Quantum Gravity. Universe 2022, 8, 116. https:// doi.org/10.3390/universe8020116 Academic Editors: Marco Danilo Claudio Torri, Christian Pfeifer and Nicoleta Voicu

Received: 7 December 2021

Accepted: 8 February 2022

Published: 12 February 2022

Publisher's Note: MDPI stays neutral with regard to jurisdictional claims in published maps and institutional affiliations.

Copyright: (c) 2022 by the authors. Licensee MDPI, Basel, Switzerland. This article is an open access article distributed under the terms and conditions of the Creative Commons Attribution (CC BY) license (https:// creativecommons.org/licenses/by/ $4.0 /)$.

\begin{abstract}
Following analogies with relativistic point particles and Schild strings, we show that the Einstein gravity and its strong coupling regime (or the Planck mass going to zero) are related to each other through a Laplace transform. The Feynman propagator of gravity in the strong coupling regime satisfies a functional diffusion equation in the three-metric space with the evolution parameter being the volume of spacetime. We conjecture that the relationship between both regimes is consistent with the existence of an evolution operator in which time is replaced by the volume of spacetime.
\end{abstract}

Keywords: quantum gravity; strong coupling limit; path integral approach

\section{Introduction}

The quantum theory of gravity has been intensively studied in the last seventy years [1] On the one hand, it is a highly complex technical problem and, on the other, a conceptual one that is not yet understood. However, the new ideas incorporated to build such a theory will undoubtedly be radically new when it happens.

Some ideas developed in the study of quantum gravity are, for example ${ }^{1}$, dissipation and determinism [5], thermodynamics [6-8], string theory [9], loop quantum gravity [10,11], or Poincaré symmetry deformations of symmetry [12-14], to mention only a few.

In this paper, we would like to study some technical and conceptual issues of quantum gravity using ideas taken from string theory. In particular, Eguchi explored a scheme in [15] for quantizing a string with finite tension from the Schild (tensionless) string [16]. The idea was applied to extended objects (relativistic membranes) $[17,18]$ and it is certainly interesting to study the case of gravity in the strong limit coupling [19,20].

We will review aforementioned cases to show that it is possible to connect theories defined in some limit of certain parameters (where the theory is usually simpler) with the theory at finite values of such parameter. The connection is provided by the Laplace transformation, which closely resembles what happens in statistical mechanics where the relation between microcanonical and canonical ensembles is considered.

The idea proposed in the present paper, is to establish a series of analogies between particles and strings and then, when these analogies are understood, to apply them to formulate a quantized gravity starting from the strong coupling regime [21-23].

The paper is organized as follows: in the next section, we discuss an approach to the quantization of particles and strings, where the Laplace transform and the strong limit have a key role. In Section 3, we apply these ideas to quantum gravity in the strong coupling regime and we analyze its possible physical implications. The last section is devoted to discussion. 


\section{Particles and Schild Strings}

In order to present these ideas, let us start by considering the Feynman propagator for a relativistic particle of mass $m$ [24]

$$
G\left[x, x^{\prime} ; m^{2}\right]=\int_{0}^{\infty} d s e^{-\frac{m^{2}}{2} s} G\left[x, x^{\prime} ; s\right],
$$

where

$$
G\left[x, x^{\prime} ; s\right]=\int \mathcal{D} x e^{-\mathcal{S}[x],}
$$

and $\mathcal{S}$ is the Euclidean action of the point particle.

This idea of introducing mass as a Laplace transform is very useful for studying the massless-massive limit transition, and, underlying this approach, are the proper-time gauge on the one hand, and, on the other hand, the boundary condition

$$
x_{1}^{\mu}(0)=x_{0}^{\mu}, \quad \text { and } \quad x_{2}^{\mu}(1)=x_{1}^{\mu},
$$

which are held fixed upon arbitrary variations. Spacetime has dimension $D$ and, therefore, index $\mu=\{0,1, \ldots, D-1\}$.

In order to calculate (2) we note that the Euclidean action is

$$
\mathcal{S}=\int_{0}^{1} d \tau \frac{1}{2 s} \dot{x}^{2}
$$

where the extra field $s(\tau)$ guarantees the invariance of the action under time reparametrization. Indeed, under the change $\tau \rightarrow \tau^{\prime}(\tau)$, the function $s$ must change as $s \rightarrow s^{\prime}\left(d \tau^{\prime} / d \tau\right)$ with $s^{\prime}=s\left(\tau\left(\tau^{\prime}\right)\right)$, to have an invariant action.

When (4) is replaced in (2), and using (3), one obtains

$$
G\left[x, x^{\prime} ; s\right]=s^{-\frac{D}{2}} e^{-\frac{(\Delta x)^{2}}{2 s}},
$$

with $\Delta x^{\mu}=x_{1}^{\mu}-x_{0}^{\mu}$. The explicit formula of the Laplace transform (1) is

$$
G\left[x, x^{\prime} ; m^{2}\right]=\int_{0}^{\infty} d s s^{-\frac{D}{2}} e^{-\frac{(\Delta x)^{2}}{2 s}-\frac{m^{2}}{2} s}
$$

the standard integral representation of the Feynman propagator in the proper-time gauge.

The appearance of the Laplace transform closely resembles the relation between microcanonical and canonical ensembles in statistical mechanics. In this analogy, what would be equivalent to the number of microcanonical states is played by $G\left[x, x^{\prime} ; s\right]$, measuring the number of configurations with a fixed parameter $s$, while the measure of the number of states with a given mass is $G\left[x, x^{\prime} ; m^{2}\right]$. The relevant point, here, is that it is possible to connect a particular limit of some parameter at which the theory is simpler, with the limit of a finite parameter. We will see how this idea is extended for strings and how can it be useful for the case of gravity.

The Feynman propagator satisfies the diffusion equation

$$
\left(\frac{\partial}{\partial s}-\frac{1}{2} \square^{2}\right) G\left[x, x^{\prime} ; s\right]=0 .
$$

From here, the connection to the WKB approach is straightforward. Indeed, applying the operator $\frac{\partial}{\partial s}-\frac{1}{2} \square^{2}$ to Equation (2) we find

$$
\left(\frac{\partial}{\partial s}-\frac{1}{2} \square^{2}\right) G\left[x, x^{\prime} ; s\right]=\left[-\frac{\partial \mathcal{S}}{\partial s}+\frac{1}{2} \square^{2} S-\frac{1}{2}(\partial \mathcal{S})^{2}\right] G\left[x, x^{\prime} ; s\right]=0,
$$


with $(\partial \mathcal{S})^{2}=\partial_{\mu} \mathcal{S} \partial_{\nu} \mathcal{S} \delta^{\mu \nu}$. The Hamilton-Jacobi equation is obtained from the last equation in $(8)$

$$
-\frac{\partial \mathcal{S}}{\partial s}=-\frac{1}{2} \square^{2} \mathcal{S}+\frac{1}{2}(\partial \mathcal{S})^{2}
$$

The term $\square^{2} \mathcal{S}$ is a quantum correction and, indeed, in the limit $\hbar \rightarrow 0$, this term cancels and we recover the Hamilton-Jacobi equation of classical mechanics.

Using this result, we turn to the study of string theory by using the following formal analogy: the role of mass corresponds to the tension $T$ in string theory. Therefore, if we can write the Feynman propagator as a Laplace transform (as before), then we can naturally make a connection with the Schild string [16].

Following the ideas above, it is natural to think that the equivalent expression to (2) is ${ }^{2}$

$$
G\left[X(\sigma), X^{\prime}(\sigma) ; T\right]=\int_{0}^{\infty} d N_{\perp} e^{-N_{\perp} T} G\left[X(\sigma), X^{\prime}(\sigma) ; N_{\perp}\right],
$$

where

$$
G\left[X(\sigma), X^{\prime}(\sigma) ; N_{\perp}\right]=\int \mathcal{D} X_{\mu}(\sigma) e^{-\mathcal{S}[X(\sigma)]},
$$

describes the Schild string dynamics with

$$
\mathcal{S}[X(\sigma)]=\int d^{2} \sigma \frac{1}{2 N_{\perp}} \dot{X}^{2}(\sigma) .
$$

Here the () and ( $)^{\prime}$ denote $\tau$ and $\sigma$ derivatives, respectively.

These above-mentioned expressions require careful physical explanation (technical results are explained in $[17,18]$ (see also [27])). First, the Schild string is the analog to a set of massless particles, and then-if the length of the string is finite, as we know-all the points of the string move at the speed of light, as implied by the constraint

$$
\mathcal{H}_{\perp}=\frac{1}{2} P^{2}(\sigma)=0
$$

However, the string also has to be invariant under reparametrizations in the worldsheet. That is, the next must be fulfilled

$$
\mathcal{H}_{1}=P_{\mu} X^{\prime \mu}=0,
$$

implying that the total Hamiltonian is

$$
H=\int d \sigma\left(N_{\perp} \mathcal{H}_{\perp}+N_{1} \mathcal{H}_{1}\right)=0,
$$

with $N_{\perp}, N_{1}$ being two Lagrange multipliers. Keeping these results in mind, we set the gauge according to

$$
\dot{N}_{\perp}=0, \quad N_{1}=0,
$$

which is the proper-time gauge [28]. In this gauge the most straightforward choice is setting $N_{\perp}$ equal to a constant, and that is what we will assume. Once this gauge is fixed, the action (12) is obtained.

With this result, the Laplace transform turns out to be a consequence of the fact that not only the ends of the string move at the speed of light (as it happens in conventional string theory) [29]. Instead, in the present case all the points on the string are moving at the speed of light.

Taking into account these considerations, the calculation of (11) is direct and the result is

$$
G\left[X(\sigma), X^{\prime}(\sigma) ; N_{\perp}\right]=s^{-\frac{D}{2}} e^{-\frac{(\Delta X(\sigma))^{2}}{2 N_{\perp}}} .
$$


The functional diffusion equation is subtle because the string is an extended object, which makes necessary the addition of new considerations.

Indeed, the presence of two "evolution" parameters, namely $\sigma$ and $\tau$, suggests that the true evolution parameter (Teichmüller one) is the area $(A)$ of the string world volume.

From the Hamiltonian point of view, the equations can be obtained as follows: the action of the string is $\mathcal{S}=\int d \tau d \sigma \mathcal{L}$, where the integral is performed in the region $\mathcal{D}$ with boundary $\partial \mathcal{D}$. Consider, now, the variation of the action under the variation of the integration domain $\mathcal{D}$, namely under the variation $\{\delta \tau(\lambda), \delta \sigma(\lambda)\}$ with $\lambda$ a parameter (usually chosen as $\lambda \in[0,1])$. The variation of the action turns out to be

$$
\delta S=\int \mathcal{L} \delta A d \lambda+\int \delta \mathcal{L} d A,
$$

with $d A=d \sigma(\lambda) d \tau(\lambda)$. The second term is the variation of the Lagrangian and it is possible to show that this term does not contribute to the variation if the action is invariant under local area transformations, which is true in the present case [15] (for a detailed discussion see also [27]). Since the action is invariant under area-preserving transformations, it changes only when the total area of $\mathcal{D}$ changes [30], and then it is possible to identify the functional derivative of the action in the previous expression with the partial derivative, that is

$$
\frac{d \mathcal{S}}{d A}=\mathcal{L}
$$

On the other hand, $S=S[A, X]$, then

$$
\frac{d \mathcal{S}}{d A}=\frac{\partial \mathcal{S}}{\partial A}+\frac{\partial \mathcal{S}}{\partial X} \frac{\partial X}{\partial A} .
$$

If we formally identify $\dot{X}=\frac{\partial X}{\partial A}$ then, by using (19) and the fact $\mathcal{H}=P \dot{X}-\mathcal{L}$, we find

$$
\mathcal{H}\left[X, \frac{\partial \mathcal{S}}{\partial X}\right]=-\frac{\partial \mathcal{S}}{\partial A}
$$

where the conjugate momentum has been identified with $\frac{\partial \mathcal{S}}{\partial X}$. The resulting equation is, therefore, the Hamilton-Jacobi equation for strings with $\mathcal{H}$, the Hamiltonian density $[31,32]$.

From the quantum point of view-and as in the case of the relativistic particle discussed above- the Feynman propagator satisfies the functional diffusion equation [15]

$$
\frac{\partial}{\partial A} G\left[X(\sigma), X^{\prime}(\sigma) ; A\right]=\frac{1}{2} \frac{\delta^{2}}{\delta X^{2}(\sigma)} G\left[X(\sigma), X^{\prime}(\sigma) ; A\right] .
$$

Equations (11) and (22) allow us to determine the Hamilton-Jacobi equation

$$
\frac{\partial}{\partial A} \mathcal{S}[X(\sigma)]=-\frac{1}{2} \frac{\delta^{2} \mathcal{S}[X(\sigma)]}{\delta X^{2}[\sigma]}+\frac{1}{2}\left(\frac{\delta \mathcal{S}[X(\sigma)]}{\delta X(\sigma)}\right)^{2} .
$$

The second derivative, namely $\delta^{2} \mathcal{S} / \delta X^{2}$, contains the quantum corrections and is the main contribution in the systematic treatment of the WKB approximation. Naturally, in the present case, Equation (23) is the Schrödinger functional equation. In the limit $\hbar \rightarrow 0$, the reparametrization constraint $P_{\mu} X^{\mu} \Psi[x(\sigma)]=-1 \frac{\delta}{\delta X^{\mu}} \Psi[x(\sigma)]$ vanishes also, as it must be due to the gauge choice.

Equations (22) and (23), and their physical implications have been intensively investigated in the past, although the derivation that we have given here is not the usual one (for extensions to p-branes, see, for example, [17]). The solutions in the WKB approximation have been considered in [33-36]. 


\section{Quantum Gravity}

Now, we go ahead with the main objective of this research and we will consider what happens with quantum gravity.

The Hamiltonian gravity action in the ADM formalism becomes

$$
\mathcal{S}=\frac{1}{16 \pi G_{N}} \int d^{4} x\left(\pi_{i j} \dot{j}^{i j}-N_{\perp} \mathcal{H}_{\perp}-N_{i} \mathcal{H}_{i}\right),
$$

where, as in the case of string theory, $N_{\perp}$ and $N_{i}$ are Lagrange multipliers, $\pi_{i j}$ is canonical momentum, $g_{i j}$ the metric tensor of the three-dimensional space, and $\mathcal{H}_{\perp}, \mathcal{H}_{i}$ are the Hamiltonian and diffeomorphism constraint, respectively. The Latin index, as usual take values $\{1,2,3\}$ and denote intrinsic quantities of the space section. $G_{N}$ is the Newton's constant.

The Hamiltonian constraints are

$$
\begin{aligned}
\mathcal{H}_{\perp} & =16 \pi G_{N} G_{i j k l} \pi^{i j} \pi^{k l}-\frac{\sqrt{g}}{16 \pi G_{N}}\left(R^{(3)}-2 \Lambda\right) \approx 0, \\
\mathcal{H}_{i} & =-2 \pi_{i ; j}^{j} \approx 0,
\end{aligned}
$$

where the supermetric is

$$
G_{i j k l}=\frac{1}{2 \sqrt{g}}\left(g_{i k} g_{j l}+g_{i l} g_{j k}-g_{i j} g_{k l}\right),
$$

and $\Lambda$ is the cosmological constant.

The coefficient $16 \pi G$ comes from the Einstein-Hilbert action. The Newton's constant $G_{N}$ has canonical dimension -2 and it can be written as the inverse of the Planck mass, that is $G_{N}^{-1}=M_{\mathrm{Pl}}^{2}$. We will use this consideration extensively below.

In order to make more explicit the analogy between string theory and gravity, let us write the Nambu-Goto action

$$
\mathcal{S}=\int d^{2} \sigma\left(P_{\mu} \dot{X}^{\mu}-N_{\perp} \mathcal{H}_{\perp}-N_{1} \mathcal{H}_{1}\right)
$$

with

$$
\begin{aligned}
\mathcal{H}_{\perp} & =\frac{1}{2}\left(\frac{1}{T} P^{2}+T X^{\prime 2}\right) \approx 0, \\
\mathcal{H}_{1} & =P_{\mu} X^{\prime \mu} \approx 0,
\end{aligned}
$$

If we compare (25) and (29) we see that the analog of a tensionless string corresponds to $M_{\mathrm{Pl}}=\frac{1}{G^{2}} \rightarrow 0$, which would be the analog of the massless spectrum in string theory. However, note that, even when the analogy is formal, it is technically interesting becauseas we will see below-it is possible to extract technical and physical information from an otherwise intractable problem.

The zero Planck mass limit is also known as the strong coupling gravity and has been studied in many contexts $[19,23,28,37,38]$ (for a recent discussion see [39] ) and the study of geometric properties is part of a very active topic, namely Carroll geometry $[40,41]$.

Another technical aspect related to Carroll's geometry is that in the limit $M_{\mathrm{Pl}} \rightarrow 0$, the spacetime is causally disconnected since the speed of a signal going from one point to a neighbor one is zero (that is, $c \rightarrow 0$ ). Parenthetically, this is the reason for the name Carroll (also note that this limit is a contraction of the Poincaré group).

Technically the limit $M_{\mathrm{Pl}} \rightarrow 0$ is equivalent to settingspatial derivatives equal to zero in the gravity theory.

Keeping these ideas in mind, we can use the proper-time gauge in gravity [28]

$$
\dot{N}_{\perp}=0, \quad N_{i}=0 .
$$


Note that the condition $\dot{N}_{\perp}=0$ establishes that, although $N_{\perp}$ does not depend on time, it can depend on spatial coordinates, but without loss of generality, we will assume $N_{\perp}$ as a constant.

The previous choice is, indeed, a good gauge choice, as we will show in what follows [23]. Consider the spacetime with topology $T \times \Sigma_{D-1}$, with $\Sigma_{D-1}$ spacelike. Generators $H_{\perp}$ and $H_{i}$ in (25) and (26) give rise to the following gauge transformations

$$
\delta N_{\perp}=\dot{\epsilon}-\left(N_{\perp} \epsilon^{i}\right)_{i}+\left(N^{i} \epsilon\right), i, \quad \delta N_{i}=\dot{\epsilon}_{i} .
$$

The variation of the action (24) under these transformations turns out to be

$$
\delta S=\int\left[\epsilon^{i} \mathcal{H}_{i}+\epsilon G_{i j k l} \pi^{i j} \pi^{k l}\right]_{t_{1}}^{t_{2}} d^{D-1} x+2 \int d t \int_{\Sigma_{D-1}} \sigma_{l} N_{k} \delta \pi^{k l} .
$$

Since the diffeomorphism constraints are satisfied, the cancellation of $\epsilon^{i} \mathcal{H}_{i}$ occurs, while the second term cancels under the assumption $\epsilon\left(t_{1}, x\right)=\epsilon\left(t_{2}, x\right)$. Note that the spatial dependence is parametric only since the limit $G_{N} \rightarrow \infty$ leaves only time dependence in $\mathcal{H}$. Finally, the second integral is zero also, due to the gauge choice.

In addition to the previous choice, it is possible to impose also the condition $\epsilon^{i}=0$, since there are not restrictions to deformations in the space directions (tangential deformations) due, again, to the strong coupling limit.

Using this last fact, the Feynman propagator becomes

$$
G\left[g_{i j}(2), g_{i j}(1) ; M_{P}\right]=\int_{0}^{\infty} d N_{\perp} e^{-M_{P}^{2} N_{\perp}} G\left[g_{i j}(2), g_{i j}(1) ; N_{\perp}\right],
$$

where

$$
G\left[g_{i j}(2), g_{i j}(1) ; N_{\perp}\right]=\int \mathcal{D} g_{i j} e^{-\int_{1}^{2} d^{4} x \frac{1}{2 N_{\perp}} G_{i j k l} \dot{g}^{i j} \dot{g} k l},
$$

where $g_{i j}(1)$ means $g_{i j}\left(\mathbf{x}, t_{1}\right)$ and the same for $g_{i j}\left(\mathbf{x}, t_{2}\right)$.

This equation is the analogue of (1) connecting the sectors with $M_{\mathrm{Pl}} \neq 0$ with $M_{\mathrm{Pl}}=0$.

It is important to emphasize that the relation (34) is an explicit prescription that relates the ultraviolet and infrared sectors and, therefore, another technical way of implementing the naturalness 't Hooft prescription [42] for quantum gravity. This, as far as we know, has not been discussed in the literature before.

Following the arguments that led us to (19), we see that we can find a Hamilton-Jacobi equation in a similar way to that of string theory but with a different physical interpretation. Indeed, in gravity the action is $\mathcal{S}=\int d^{4} x \mathcal{L}$, with $\mathcal{L}$ the Einstein-Hilbert Lagrangian. However, the invariance under reparametrization suggests that we can use the volume of spacetime as a parameter of evolution, so, by analogy with (19), we have

$$
\frac{d \mathcal{S}}{d V}=\mathcal{L}
$$

and the Hamilton-Jacobi equation turns out to be (21) with the replacement $A \rightarrow V$ being $V$ the volume of the integration domain. This assumption also follows the Moser theorem, extended to non-compact manifolds in [43], and it remains valid for volume-invariant diffeomrphisms (see [23] for a discussion in the $2+1$ case).

However (35) implies

$$
-\frac{\partial \mathcal{S}}{\partial V}=G^{i j k l} \frac{\delta \mathcal{S}}{\delta g^{i j}} \frac{\delta \mathcal{S}}{\delta g^{k l}}-\frac{\delta}{\delta g^{i j}}\left(G^{i j k l} \frac{\delta \mathcal{S}}{\delta g^{k l}}\right),
$$

which is, on the one hand, a much more complicated equation and, on the other, has different physical implications than those we will point out below. 
The first comment is that (34) is a causal relationship between two regions with different scales. However, (34) and the existence of the Laplace transform are consistent with a state evolution operator, but with $V$ playing the role of time in quantum gravity.

We also emphasize that the Laplace transform (21) is an unexpected result because, although it was obtained following the Feynman construction, in the case of gravity, it turns out to be a relationship that connects very different energy sectors.

\section{Discussion}

Following an analogy with string theory (and point-relativistic particles), we have written the Feynman propagator between two geometries as the Laplace transform of the propagator describing quantum gravity in the strong coupling limit.

Additionally, like the aforementioned cases, the strong coupling limit propagator satisfies a functional diffusion equation, with the four-dimensional volume playing the role of a time parameter.

This approach seems to be particularly suitable for treating the pure time evolution of the metric, as, for example, in the case of quantum cosmology. In fact, for such a case, the action in (35) is proportional to the three-dimensional volume and the integral contains only the scale factor. To gain an insight on this, consider the three-dimensional metric $g=a^{2}(t) \operatorname{diag}(1,1,1)$, then

$$
G\left[a\left(t_{2}\right), a\left(t_{1}\right) ; N_{\perp}\right]=\int \mathcal{D} a e^{3 V \int_{t_{1}}^{t_{2}} \frac{1}{N_{\perp}} \frac{\dot{a}^{2}}{a^{5}} d t}
$$

The action in the exponential turns out to be the action in the strong coupling limit. Various issues, such as solutions of Einstein's equations in the strong coupling regime and explicit quantum formulas have not been written. These and other topics will be the purpose of a future research work.

Author Contributions: All authors contributed equally to this work. All authors have read and agreed to the published version of the manuscript.

Funding: This research was supported by DICYT 042131GR (J.G.) and 041931MF (F.M.) and by the U.S. Department of Energy under the Award No. DE-SC0020250 (N.T-A.).

Institutional Review Board Statement: Not applicable.

Informed Consent Statement: Not applicable.

Acknowledgments: One of us (J.G.) thanks the Alexander von Humboldt Foundation by support.

Conflicts of Interest: The authors declare no conflict of interest.

\section{Notes}

1 The literature about this topic is extraordinarily vast, and it is impossible (and surely unpractical) to give a proper bibliographical citation to all works on the subject. We limit ourselves only to original references and reviews. See, for example [2,3]. For a phenomenology approach see [4].

2 We will adopt the standard notation $s=N_{\perp}$ for the rest of the text (see, for example $[25,26]$ ). Then, the proper-time gauge reads $N_{\perp}=$ constant.

\section{References}

1. Kiefer, C. Quantum Gravity, 2nd ed.; Oxford University Press: New York, NY, USA, 2007.

2. Carlip, S.; Chiou, D.W.; Ni, W.T.; Woodard, R. Quantum Gravity: A Brief History of Ideas and Some Prospects. Int. J. Mod. Phys. D 2015, 24, 1530028. [CrossRef]

3. Stachel, J. The Early History of Quantum Gravity (1916-1940). In Black Holes, Gravitational Radiation and the Universe: Essays in Honor of C.V. Vishveshwara; Iyer, B.R., Bhawal, B., Eds.; Springer: Dordrecht, The Netherlands, 1999; pp. 525-534. [CrossRef]

4. Addazi, A.; Alvarez-Muniz, J.; Batista, R.A.; Amelino-Camelia, G.; Antonelli, V.; Arzano, M.; Asorey, M.; Atteia, J.-L.; Bahamonde, S.; Bajardi, F.; et al. Quantum gravity phenomenology at the dawn of the multi-messenger era-A review. arXiv 2021, arXiv:2111.05659.

5. 't Hooft, G. Quantum gravity as a dissipative deterministic system. Class. Quant. Grav. 1999, 16, 3263-3279. [CrossRef] 
6. $\quad$ Bekenstein, J.D. Black holes and the second law. Lett. Nuovo C. 1972, 4, 737-740. [CrossRef]

7. Bekenstein, J.D. Black holes and entropy. Phys. Rev. D 1973, 7, 2333-2346. [CrossRef]

8. Bekenstein, J.D. Generalized second law of thermodynamics in black hole physics. Phys. Rev. D 1974, 9, 3292-3300. [CrossRef]

9. Aharony, O.; Gubser, S.S.; Maldacena, J.M.; Ooguri, H.; Oz, Y. Large N field theories, string theory and gravity. Phys. Rep. 2000, 323, 183-386. [CrossRef]

10. Ashtekar, A.; Lewandowski, J. Background independent quantum gravity: A Status report. Class. Quant. Grav. 2004, 21, R53. [CrossRef]

11. Rovelli, C. Loop Quantum Gravity. Living Rev. Relativ. 2008, 11, 5. [CrossRef]

12. Lukierski, J.; Ruegg, H.; Nowicki, A.; Tolstoi, V.N. Q deformation of Poincare algebra. Phys. Lett. B 1991, 264, 331-338. [CrossRef]

13. Ballesteros, A.; Gubitosi, G.; Gutiérrez-Sagredo, I.; Herranz, F.J. Curved momentum spaces from quantum groups with cosmological constant. Phys. Lett. B 2017, 773, 47-53. [CrossRef]

14. Addazi, A.; Marcianò, A. A modern guide to $\theta$-Poincaré. Int. J. Mod. Phys. A 2020, 35, 2042003. [CrossRef]

15. Eguchi, T. New Approach to the Quantized String Theory. Phys. Rev. Lett. 1980, 44, 126. [CrossRef]

16. Schild, A. Classical Null Strings. Phys. Rev. D 1977, 16, 1722. [CrossRef]

17. Gamboa, J.; Ruiz-Altaba, M. Path integral approach to membranes. Phys. Lett. B 1988, 205, 245-249. [CrossRef]

18. Gamboa, J.; Ramirez, C.; Ruiz-Altaba, M. Field Theory of Null Strings and $p$-branes. Phys. Lett. B 1989, 231, 57-60. [CrossRef]

19. Pilati, M. Strong Coupling Quantum Gravity. 1. Solution in a Particular Gauge. Phys. Rev. D 1982, 26, 2645. [CrossRef]

20. Henneaux, M.; Pilati, M.; Teitelboim, C. Explicit Solution for the Zero Signature (Strong Coupling) Limit of the Propagation Amplitude in Quantum Gravity. Phys. Lett. B 1982, 110, 123-128. [CrossRef]

21. Gamboa, J. A Model of two-dimensional quantum gravity in the strong coupling regime. Phys. Rev. Lett. 1995, 74, 1900-1903. [CrossRef]

22. Gamboa, J. On the initial singularity problem in two-dimensional quantum cosmology. Phys. Rev. D 1996, 53, 6991-6994. [CrossRef]

23. Gamboa, J.; Mendez, F. Quantum gravity at very high-energies. Nucl. Phys. B 2001, 600, 378-386. [CrossRef]

24. Feynman, R.P. Mathematical formulation of the quantum theory of electromagnetic interaction. Phys. Rev. 1950, 80, 440-457. [CrossRef]

25. Nambu, Y. The use of the Proper Time in Quantum Electrodynamics. Prog. Theor. Phys. 1950, 5, 82-94. [CrossRef]

26. Schwinger, J.S. The Theory of quantized fields. 1. Phys. Rev. 1951, 82, 914-927. [CrossRef]

27. Ogielski, A.T. Comments on the 'Planar Time' Dynamics of Strings. Phys. Rev. D 1980, 22, 2407. [CrossRef]

28. Teitelboim, C. Quantum Mechanics of the Gravitational Field. Phys. Rev. D 1982, 25, 3159. [CrossRef]

29. Kalb, M.; Ramond, P. Classical direct interstring action. Phys. Rev. D 1974, 9, 2273-2284. [CrossRef]

30. Moser, J. On the volume elements on a manifold. Trans. Am. Math. Soc. 1969, 120, 286-294. [CrossRef]

31. Hosotani, Y. Hamilton-Jacobi Formalism and Wave Equations for Strings. Phys. Rev. Lett. 1985, 55, 1719. [CrossRef]

32. Luscher, M. The Area Law as a Consequence of the Loop Wave Equation. Phys. Lett. B 1980, 90, 277-279. [CrossRef]

33. Luscher, M.; Symanzik, K.; Weisz, P. Anomalies of the Free Loop Wave Equation in the WKB Approximation. Nucl. Phys. B 1980, 173, 365. [CrossRef]

34. Alvarez, O. Theory of Strings with Boundaries: Fluctuations, Topology, and Quantum Geometry. Nucl. Phys. B 1983, 216, 125-184. [CrossRef]

35. Migdal, A.A. Loop Equations and 1/N Expansion. Phys. Rep. 1983, 102, 199-290. [CrossRef]

36. Vassilevich, D.V. Heat kernel expansion: User's manual. Phys. Rep. 2003, 388, 279-360. [CrossRef]

37. Pilati, M. Strong coupling quantum gravity. 2. Solution without gauge fixing. Phys. Rev. D 1983, 28, 729. [CrossRef]

38. Gamboa, J.; MacKenzie, R.; Méndez, F. Gravitons in the Strong-Coupling Regime. arXiv 2020, arXiv:2010.12966.

39. Brax, P.; Rizzo, L.A.; Valageas, P. Ultralocal models of modified gravity without kinetic term. Phys. Rev. D 2016, $94,044027$. [CrossRef]

40. Lévy-Leblond, J.M. Une nouvelle limite non-relativiste du groupe de Poincaré. Ann. Phys. Theor. 1965, 3, 1-12.

41. Bacry, H.; Levy-Leblond, J. Possible kinematics. J. Math. Phys. 1968, 9, 1605-1614. [CrossRef]

42. 't Hooft, G. Under the Spell of the Gauge Principle, 1st ed.; World Scientific: London, UK, 1994.

43. Green, R.E.; Shiohama, K. Diffeomorphisms and volume-preserving embeddings of noncompact manifolds. Trans. Am. Math. Soc. 1979, 255, 403. [CrossRef] 